\title{
Prevalence of Metabolic Syndrome and Its Individual Components in Patients with AMI and Consideration of Obesity Markers among These Patients
}

\author{
Dr. Sanjay Sarkar ${ }^{1}$, Dr. Seema Mondal $^{2}$, Dr. Tushar Kanti Saha ${ }^{3}$ \\ ${ }^{I}\left(\right.$ RMO, B.S. Medical College, Bankura, India) ${ }^{2}$ (Asst. Prof., Burdwan Medical College, India) \\ ${ }^{3}$ (Asst. Prof., NRS Medical College, Kolkata, India)
}

\begin{abstract}
Background: Many studies have estimated the prevalence of Metabolic Syndrome [MS] and also its association with type II Diabetes and cardiovascular disease. But its prevalence and association with acute cardiovascular events like Acute Myocardial Infarction [AMI] remained largely unexplored.

Objectives: (1) To determine the prevalence of the metabolic syndrome in patients with AMI; (2) To find out the association of the individual component of the metabolic syndrome with AMI. (3) To study the association of obesity markers like waist circumference [WC], Body Mass Index [BMI] and Waist Hip Ratio [WHR] with AMI.

Study Design: Hospital based cross-sectional case control study.

Methods: The study was conducted in a Medical College of Kolkata, West Bengal. Applying National Cholesterol Education Program Adult Treatment Panel III (NCEP-ATP III) criteria and evaluated subjects for the 5 component conditions of the metabolic syndrome: insulin resistance, abdominal obesity based on waist circumference, hypertriglyceridemia, low HDL cholesterol (HDL-C), and hypertension, as well as the full syndrome, defined by the presence of at least 3 of the 5 conditions.
\end{abstract}

Statistical Analysis: Student's t test and Chi-square test.

Results: Among the 76 AMI patients, 47.36\% male AMI cases and $46.87 \%$ of female AMI cases had MS and both are statistically significant when compared with controls. Among the 5 component conditions of MS, 4 components like insulin resistance, hypertension, hypertriglyceridemia and low HDL cholesterol were more prevalent in cases in both the sex groups compared with controls and the results were statistically significant. Only one exception obesity measured by high WC [p>0.05] which was not independently related to MS. Among the component conditions insulin resistance had the highest OR in men and low HDL had the highest OR in women. Among other obesity markers the association of BMI with MI was not statistically significant whereas central obesity measured by WHR of $>0.09$ were significantly related to MI in both male and female group. Conclusion: The study indicates that prevalence of MS and its individual component conditions are significantly related to AMI. Considering the marker of obesity waist circumference and BMI were not significantly related to AMI whereas WHR is significantly related to AMI. Maybe this is the time when we should look upon to derive the cut off points for obesity with consideration specifically related to Asian Indians.

Keywords: Metabolic syndrome, acute myocardial infarction

\section{Introduction}

The term Metabolic Syndrome was first used by J. P. Camus (1966) who revealed the connection between diabetes mellitus, hyperlipoproteinemia and gout with high cardiovascular risk. ${ }^{(1)}$ Twenty years later Raeven described Syndrome X (diabetes mellitus, dyslipidemia: hypertriglyceridemia with the reduction of HDL cholesterol, arterial hypertension and insulin resistance). ${ }^{(2)}$ In 1999 WHO introduced the name Metabolic Syndrome $\mathrm{X}^{(3,4)}$ and established it as a distinct clinical entity. A group of metabolic and physiological risk factors with specific cut off values which are linked to cardiovascular disorders (CVD) has been variously termed as the insulin resistance syndrome, the metabolic syndrome, the dysmetabolic syndrome. ${ }^{(5-12)}$ The metabolic syndrome is one of the major public health issues of this century. The presence of MS is associated with an increased risk of coronary heart disease. ${ }^{(13,14)}$ Proposed definitions of the metabolic syndrome have differed with respect to components and their cut points. In the present study, we have examined the prevalence and association of metabolic syndrome and its individual components in patients admitted with myocardial infarction (MI).

Obesity is largely prevalent in our country. Studies are ongoing to validate the cut off values, used by the international bodies, among Asian Indians. This effort is to identify patients at risk for diabetes and CVD due to overweight or obesity. In our study we have also tried to see the prevalence and significance of association of WHR and BMI in patients with AMI. 


\section{Materials and Methods}

This hospital-based, cross-sectional study was conducted in the Department of Cardiology, Calcutta National Medical College and Hospital (CNMC\&H), Kolkata, West Bengal. The study was carried out from March 2004 to July 2005. 76 patients admitted in the coronary care unit, who were diagnosed as having AMI as per the WHO criteria, were included in the study. Blood samples were drawn at admission for creatine kinasetotal and creatine kinase- MB isoenzyme. The following morning, samples for triglyceride and HDL-C were collected, and on day 3, a sample for estimation of blood glucose level was collected. All the biochemical tests are done at the biochemistry department of CNMC\&H. Waist-circumference and blood pressure were recorded in all subjects.

We have used the National Cholesterol Education Program Adult Treatment Panel III (NCEP-ATP III) recommendations for defining the MS which consists of any three of the following 5 component conditions: hypertriglyceridemia (HTG), low HDL cholesterol (HDL-C), hypertension (HTN), abdominal obesity, and insulin resistance (IR). ${ }^{(15)}$ IR was defined as fasting glucose $\geq 110 \mathrm{mg} / \mathrm{dL}$ or by a self-report of current use of insulin or oral hypoglycemics. HTN was defined as systolic blood pressure $\geq 130 \mathrm{~mm} \mathrm{Hg}$. HTN may also can be diagnosed if diastolic blood pressure $\geq 85 \mathrm{~mm} \mathrm{Hg}$ or by a self-report of current use of anti-HTN medication. HTG was identified based on triglycerides $\geq 150 \mathrm{mg} / \mathrm{dL}$. Low HDL-C was identified by HDL cholesterol $<40$ $\mathrm{mg} / \mathrm{dL}$ in men or $<50 \mathrm{mg} / \mathrm{dL}$ in women. High WC was defined as a WC $>102 \mathrm{~cm}$ in men or $>88 \mathrm{~cm}$ in women. The present study was based on a subset of subjects aged 20 to 89 years. The WC of subjects were measured using a measuring tape positioned at the high point of the iliac crest. The measurement was made at minimal respiration to the nearest $0.1 \mathrm{~cm}$, with the tape snug but not compressing the skin. Subject age, race, and cigarette smoking outcome were collected in a structured interview. A minimum age cut off of 20 years is consistent with the NCEP-ATP-III definition of young adult.

To determine if there is a statistically significant difference we used t-test for independent samples. Data was analyzed using the Student's t test and the Chi-square test.

\section{Results and Analysis}

Seventy six [76] patients with a confirmed diagnosis of AMI were included in the study by selecting randomly. Among the patients 38 were male and 32 were female. There were 38 male and 32 female age and sex matched controls chosen randomly. Among the 38 male cases 18 had MS [47.36\%] compared to 3 persons who had MS out of 38 male controls. Statistical analysis showed that the difference was statistically significant [ $p<0.001]$. Among the 32 female cases 15 had MS [46.87\%] compared to 2 persons who had MS out of 32 controls. Statistical analysis reveals that the difference is also statistically significant $[\mathrm{p}<0.001]$. The individual component conditions of MS were also compared among the cases of AMI and controls in both sexes. Also the other anthropometric markers of obesity eg. BMI [which is a measure of generalized obesity of body] and WHR [a measure of central obesity of the body] are compared. Summary of the results are tabulated in table 1 and table 2. The prevalence of each component conditions (insulin resistance, hypertension, abdominal obesity measured by waist circumference, hypertriglyceridemia and low HDL cholesterol) are more common in cases in both the male and female groups compared with controls. All results are statistically significant except for abdominal obesity measured by high waist circumference [p>0.05] which was not independently related to the prevalent disease.

Table-1 shows the prevalence of the component conditions and the full metabolic syndrome among subjects with and without prevalent disease, both in male and females. There is no significant difference in prevalence of MS among male and female cases of AMI.

Table-2 shows the sex specific association of MI with the full metabolic syndrome and its component conditions, adjusted for age, sex, race, and smoking status. The metabolic syndrome was significantly associated with MI in both men (OR, 10.50; 95\% CI,) and women (OR, 13.24; 95\% CI,). All the component conditions of metabolic syndrome were significantly associated with MI except abdominal obesity, measured by waist circumference.

Among the component conditions insulin resistance had the highest OR in men and low HDL had the highest OR in women. An interesting finding of other obesity markers was revealed in this study. When analysing the association of BMI with MI, our study reveal that their relation were not statistically significant. But when central obesity was measured by waist hip ratio of $>0.09$ our study revealed that they were significantly related to MI and that holds true in both male and female group.

\section{Discussion}

The present study was a hospital based comparative case control study conducted in National Medical College and Hospital, a tertiary care hospital of Calcutta, West Bengal, serving an urban and suburban population. In the present study, we found that the metabolic syndrome was significantly associated with AMI in both sexes. These findings suggest that the metabolic syndrome has clinical utility in identifying patients at 
increased risk of AMI. Similar findings have been noted by John K. Ninomiya in his analysis of Third National Health and Nutrition Examination Survey where MS is significantly associated with AMI. ${ }^{(16)}$ a few similar studies have reported higher cardiovascular risk in subjects with $\mathrm{MS}^{\cdot(17,18)}$

Among the components of MS all are novel risk factors for AMI and among them hypertension, insulin resistance, low HDL and high triglyceride are significantly present in patients of AMI in both the sexes. But there is lack of association between abdominal obesity (high WC) and prevalent disease. Also when taking into consideration of other obesity markers, statistical significance do not reach for body mass index [BMI] but the waist hip ratio [WHR] reached statistical significance. This differs from some previous studies that have reported linear relationships between coronary heart disease and either BMI or WHR. ${ }^{(19)}$ Though the recent INTERHEART study showed that waist-to-hip ratio was a much better predictor of CVD events than BMI. ${ }^{(20)}$ This finding may reflect an indirect effect of high WC through other components of the syndrome or may be due to higher cut off values used to determine obesity. It must be emphasized that While it is clear that obesity measured by any index almost always correlates with cardiovascular disease risk (CVD) risk factors, there are differences in the relationship of these anthropometric measures and CVD risk factors in different ethnic groups. ${ }^{(21)}$

It is seen that Asian Indians have an increased predisposition not only to diabetes ${ }^{(22)}$ but also to premature coronary artery disease. ${ }^{(23)}$ This has been attributed to the so called 'Asian Indian Phenotype' of obesity characterized by less of generalized obesity as measured by body mass index (BMI) but greater central body obesity as measured by greater WC and WHR. ${ }^{(24,25)}$ Obesity is a major driver for the widely prevalent metabolic syndrome and type 2 diabetes mellitus (T2DM) in Asian Indians in India and those residing in other countries. In this study the lack of association of BMI and WC with the prevalent disease, AMI, may be due to the use of higher cut off values used in this study, which have used the cut offs based on Caucasian population. This study also emphasizes the need for determining Asian Indian specific cut offs for obesity. Based on percentage body fat and morbidity data, limits of normal BMI are narrower and lower in Asian Indians than in white Caucasians.

We also like to emphasize that large scale prospective studies are required to know the influence of MS on the outcome or case fatality rates in AMI.

\section{Conclusion}

So we like to conclude that our study had found significant association between the metabolic syndrome and AMI. In addition, with the exception of high WC, all of the component conditions of the metabolic syndrome were significantly associated with AMI. These findings clinches the importance of the metabolic syndrome as a significant risk factor for cardiovascular disease. This reaffirms the need to develop stringent strategies for controlling this syndrome and also its individual components. This study also indicates the need to develop Asian Indian specific cut off values for determining obesity.

[1] Rudolph H., Forschung und Praxis, 174 (1994) 3.

[2] Reaven GM. Role of insulin resistance in human disease. Diabetes. 1988;37:1595- 607..

[3] Alberi KGMM, Zimmet PZ, for the WHO Consultation. Definition, diagnosis and classification of diabetes mellitus and its complications, part 1: diagnosis and classification of diabetes mellitus, provisional report of a WHO consultation. Diabet Med. 1998;15:539-553.

[4] Piatti P., G. Fragasso, L. D. Monti, A. Caumo, C. Van Phan, G. Valsecchi, S. Costa, E. Focheasto, G. Pozza, A. E. Pontiroli, S. Chierchia, J. Am. Coll. Cardiol., 34 (1999) 1452.

[5] Deedwania PC. The deadly quartet revisited. Am J Med. 1998;105(suppl 1A):1S-3S.

[6] Fagan TC, Deedwania PC. The cardiovascular dysmetabolic syndrome. Am J Med. 1998;105(suppl 1A):77S-82S.

[7] Stern MP. Diabetes and cardiovascular disease. The "common soil" hypothesis. Diabetes 1995;44:369-74.

[8] Laaksonen DE, Lakka HM, Niskanen LK, Kaplan GA, Salonen JT, Lakka TA. Metabolic syndrome and development of diabetes mellitus: application and validation of recently suggested definitions of the metabolic syndrome in a prospective cohort study. Am J Epidemiol. 2002;156:1070-7.

[9] Grundy SM, Cleeman JI, Daniels SR, Donato KA, Eckel RH, Franklin BA, et al. Diagnosis and management of the metabolic syndrome: an American Heart Association/National Heart, Lung, and Blood Institute Scientific Statement. Circulation. 2005;112:2735-52.

[10] Lindsay RS, Howard BV. Cardiovascular risk associated with the metabolic syndrome. Curr Diab Rep. 2004;4:63-8.

[11] Stern MP, Williams K, Gonza'lez-Villalpando C, Hunt KJ, Haffner SM. Does the metabolic syndrome improve identification of individuals at risk of type 2 diabetes and/or cardiovascular disease? Diabetes Care. 2004;27:2676-81.

[12] Alberti KG, Zimmet P, Shaw J. The metabolic syndrome: a new worldwide definition. Lancet. 2005;366:1059-62.

[13] Wilson PW, Kannel WB, Silbershatz H, D’Agostino RB. Clustering of metabolic factors and coronary heart disease. Arch Intern Med 1999;159:1104-9.

[14] Isomaa B, Almgren P, Tuomi T, et al. Cardiovascular morbidity and mortality associated with the metabolic syndrome. Diabetes Care 2001;24:683-9.

[15] Expert Panel on Detection, Evaluation, and Treatment of High Blood Cholesterol in Adults. Executive summary of the third report of the National Cholesterol Education Program (NCEP) expert panel on detection, evaluation and treatment of high blood cholesterol in adults (Adult Treatment Panel III). JAMA. 2001;285:2486-2497. 
[16] John K. Ninomiya, Gilbert L'Italien, Michael H. Criqui, Joanna L. Whyte, Anthony, Gamst and Roland S. Chen et al. Association of the Metabolic Syndrome With History of Myocardial Infarction and Stroke in the Third National Health and Nutrition Examination Survey. Circulation 2004;109:42-46.

[17] Wilson PW, Kannel WB, Silbershatz H, D'Agostino RB. Clustering of metabolic factors and coronary heart disease. Arch Intern Med 1999;159:1104-9.

[18] Isomaa B, Almgren P, Tuomi T, et al. Cardiovascular morbidity and mortality associated with the metabolic syndrome. Diabetes Care 2001;24:683-9.

[19] Daly P, Solomon C, Manson J. Risk modification in the obese patient. In: Manson J, Ridker P, Gaziano J, et al, eds. Prevention of Myocardial Infarction. New York: Oxford University Press; 1996:203-240.

[20] Yusuf S, Hawken S, Ounpuu S, Bautista L, Franzosi MG, Commerford P, et al. Obesity and the risk of myocardial infarction in 27000 participants from 52 countries: a case-control study. The Lancet 2005; 366: 1640-9.

[21] Gupta R, Rastogi Priyanka, Sarna M, et al. Body mass index, waist-size, waist-hip ratio and cardiovascular risk factors in urban subjects. J Assoc Physicians India. 2007;55:621-27.

[22] Wild S, Roglic G, Green A, Sicree R, King H. Global prevalence of diabetes, estimates for the year 2000 and projections for 2030. Diabetes Care 2004;27:1047-53.

[23] Reddy KS, Yusuf S. Emerging Epidemic of Cardiovascular Disease in Developing Countries. Circulation 1998;97:596- 601.

[24] Joshi R. Metabolic syndrome - Emerging clusters of the Indian Phenotype. J Assoc Physicians India 2003;51:445-6.

[25] Deepa R, Sandeep S, Mohan V. Abdominal obesity, visceral fat and type 2 diabetes - 'Asian Indian phenotype'. In: Mohan V, Gundu Rao, eds. Type 2 diabetes in South Asians: Epidemiology, risk factors and prevention. Jaypee Brothers Medical publishers, 2006; 138-52.

Table - 1: Prevalence of the component conditions and the full metabolic syndrome among cases of myocardial infarction and controls

\begin{tabular}{|c|c|c|c|c|}
\hline & Male cases & Male controls & Female cases & Female controls \\
\hline Numbers & 38 & 38 & 32 & 32 \\
\hline Average age & 51.557 .77 & 50.877 .82 & 52.878 .50 & 52.818 .53 \\
\hline MS \% [N] & $47.36 \%[18 / 38]$ & $13.15 \%[3 / 38]$ & $46.87 \%[15 / 32]$ & $9.37 \%[2 / 32]$ \\
\hline \multicolumn{5}{|l|}{ Component s of MS } \\
\hline Abdominal obesity[WC] & $18.42 \%[7 / 38]$ & $7.89 \%[3 / 38]$ & $15.62 \%[5 / 32]$ & $6.25 \%[2 / 32]$ \\
\hline High TG & $42.10 \%[16 / 38]$ & $15.78 \%[6 / 38]$ & $43.75 \%[14 / 32]$ & $18.75 \%[6 / 32]$ \\
\hline Low HDL & $52.63 \%[20 / 38]$ & $15.78 \%[6 / 38]$ & $62.50 \%[20 / 32]$ & $18.75 \%[6 / 32]$ \\
\hline Hypertension & $34.21 \%[13 / 38]$ & $7.89 \%[3 / 38]$ & $31.25 \%[10 / 32]$ & $6.25 \%[2 / 32]$ \\
\hline Insulin resistance & $31.58 \%[12 / 38]$ & $5.26 \%[2 / 38]$ & $34.37 \%[11 / 32]$ & $9.37 \%[3 / 32]$ \\
\hline \multicolumn{5}{|l|}{$\begin{array}{l}\text { Other markers of } \\
\text { abdominal obesity }\end{array}$} \\
\hline WHR & $36.84 \%[14 / 38]$ & $7.90 \%[3 / 38]$ & $28.12 \%[9 / 32]$ & $6.25 \%[2 / 32]$ \\
\hline BMI & $15.80 \%[6 / 38]$ & $5.26 \%[2 / 38]$ & $12.50 \%[4 / 32]$ & $6.25 \%[2 / 32]$ \\
\hline
\end{tabular}

Table-2: Cross-Sectional Association of MI With the Metabolic Syndrome and Component Conditions by gender

\begin{tabular}{|c|c|c|c|c|c|c|}
\hline \multirow[b]{2}{*}{ Syndrome components } & \multicolumn{3}{|c|}{ MI [male] } & \multicolumn{3}{|c|}{ MI [ female] } \\
\hline & OR & $95 \% \mathrm{CI}$ & $\mathrm{P}$ value & OR & $95 \% \mathrm{CI}$ & $P$ value \\
\hline Waist circumference & 2.63 & $0.62-11.07$ & $>0.05$ & 2.73 & $0.49-15.02$ & $>0.05$ \\
\hline High triglyceride & 3.80 & $1.31-11.47$ & $<0.01$ & 3.11 & $1.04-9.28$ & $<0.01$ \\
\hline Decreased HDL-C & 5.93 & $2.01-17.44$ & $<0.001$ & 5.92 & 2.01-16.44 & $<0.001$ \\
\hline Hypertension & 6.07 & $1.56-23.54$ & $<0.01$ & 6.43 & $1.30-31.73$ & $<0.02$ \\
\hline Insulin resistance & 8.31 & $1.71-40.32$ & $<0.01$ & 4.75 & $1.20-18.74$ & $<0.05$ \\
\hline $\begin{array}{l}\text { Metabolic syndrome } \\
\text { Other markers of abdominal obe }\end{array}$ & 10.50 & $2.75-40.10$ & $<0.001$ & 11.74 & $2.45-56.17$ & $<0.001$ \\
\hline Waist hip ratio [ $\mathrm{W}: \mathrm{H}]$ & 6.81 & $1.76-26.27$ & $<0.01$ & 5.58 & $1.12-27.90$ & $<0.05$ \\
\hline BMI [ body mass index] & 3.38 & $0.64-17.92$ & $>0.05$ & 2.11 & $0.36-12.32$ & $>0.05$ \\
\hline
\end{tabular}

